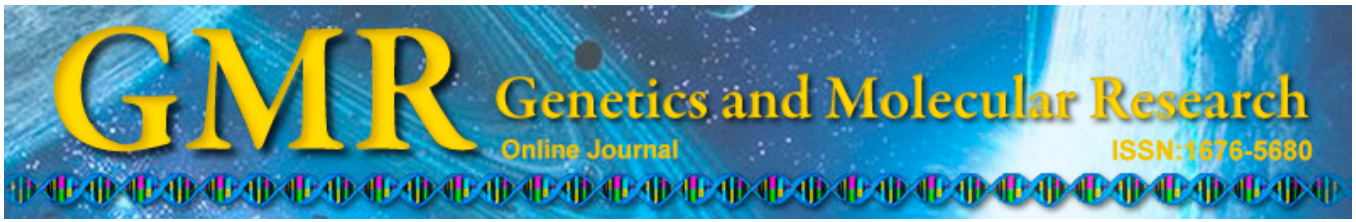

\title{
Expression divergence of FRUITFULL homeologs enhanced pod shatter resistance in Brassica napus
}

\author{
P.F. Peng ${ }^{1}$, Y.C. Li ${ }^{1}$, D.S. Mei ${ }^{1}$, J. Colasanti ${ }^{2}$, L. Fu ${ }^{1}$, J. Liu ${ }^{1}$, Y.F. Chen ${ }^{1}$ \\ and $\mathrm{Q} . \mathrm{Hu}^{1}$ \\ ${ }^{1}$ Key Laboratory of Biology and Genetic Improvement of Oil Crops, \\ Ministry of Agriculture, \\ Oil Crops Research Institute of the Chinese Academy of Agricultural Sciences, \\ Wuhan, Hubei, China \\ ${ }^{2}$ Department of Molecular and Cellular Biology, University of Guelph, \\ Guelph, Ontario, Canada \\ Corresponding author: Q. Hu \\ E-mail: huqiong@oilcrops.cn \\ Genet. Mol. Res. 14 (1): 871-885 (2015) \\ Received February 17, 2014 \\ Accepted October 11, 2014 \\ Published February 2, 2015 \\ DOI http://dx.doi.org/10.4238/2015.February.2.11
}

\begin{abstract}
To improve pod shatter resistance in the important oilseed crop Brassica napus, the phenotypic diversity of $B$. napus was tested using 80 B. napus varieties for pod shatter resistance by a random impact test. Among these varieties, R1-1 was identified as resistant, while R2, 8908B was susceptible to shatter. To understand the molecular basis for this phenotypic difference based on the candidate gene approach, $B$. napus FRUITFULL (FUL) homologs were identified and characterized. Two FUL loci in the A and $\mathrm{C}$ genomes of $B$. napus were identified. In the susceptible variety, both BnaA.FUL and BnaC.FUL were expressed in the same tissues. However, the expression level of BnaC.FUL differed in varieties with different pod shatter resistance. In the most resistant variety, R1-1, only BnaA.FUL was expressed, while BnaC.FUL was silenced. Therefore, the functional divergence and differing expression
\end{abstract}


of BnaX.FUL homeologs may significantly affect phenotypic variation, which is an important consequence of allopolyploid evolution. This expression level divergence may be useful for selecting pod shatter resistant lines through marker-assisted selection in B. napus-breeding programs.

Key words: Allopolyploid evolution; Expression divergence; FUL homeolog; Oilseed rape; Pod shattering

\section{INTRODUCTION}

The important oil crop Brassica napus, commonly known as oilseed rape, provides healthy edible oil for human consumption and may also be important as a biodiesel crop. At harvest, pod shattering can easily increase yield losses, particularly when seeds are harvested through mechanical means such as combines. Therefore, reducing pod shattering is a major objective in B. napus-breeding programs. However, little is known regarding pod shattering in oilseed rape because the pod shatter process is very complex, involving plant growth, silique development, hormone effects, and other processes (Child et al., 2003; Wang et al., 2007). In addition, the lack of oilseed rape germplasms resistant to pod shattering has limited this research (Wang et al., 2007).

The pods of $B$. napus contain 2 valves separated by the replum, which is in the center of the pod that extends along the 2 valves. Pod shattering involves the dehiscence zones (DZ), which are different from the valve edge cells and are located between the valve and the replum. In Arabidopsis, the DZ can be divided into 2 layers, a separation layer joined to the replum and a lignified layer joined to the valve (Lewis et al., 2006). DZ cells degrade and separate, which contributes to silique opening when the pods are mature.

Advances in molecular biology and genomics have enabled the development of oilseed rape lines resistant to pod shattering through genetic engineering or marker-assisted selection. Currently, with the development of whole-genome sequencing and functional gene identification methods in model plants, the candidate gene approach has been shown to be efficient for identifying key functional genes involved in specific processes (Salentijn et al., 2007). Oilseed rape has a high degree of genomic sequence identity to Arabidopsis thaliana, indicating its close relationship with this model plant species. Therefore, $A$. thaliana can be used to formulate hypotheses regarding gene function in $B$. napus. Homologs of $A$. thaliana genes typically have similar functions in B. napus (Chai et al., 2010). As functional genes have been identified in $A$. thaliana (Bolle et al., 2011), the use of these genes to identify functional $B$. napus genes with agronomic importance has become increasingly feasible.

Studies of $A$. thaliana have identified a complex regulatory network involved in pod shattering, including identification of some transcription factors involved in the development of valve margins. For example, INDEHISCENT, which is expressed in the 2 layers, is required for the development of valve margins (Liljegren et al., 2004), and ALCATRAZ (ALC) is involved in the development of the separation layers (Rajani and Sundaresan, 2001). Two MADS-box genes, SHATTERPROOF1 and SHATTERPROOF2, promote the expression of INDEHISCENT and ALC, facilitating the lignification of DZ cells (Liljegren et al., 2000). The valves and the replum failed to detach in the shatterproof 1 shatterproof 2 double mutant. FRUITFULL (FUL) regulates cell elongation and differentiation of the valve and negatively 
regulates the expression of the SHPS (Gu et al., 1998; Ferrandiz et al., 2000). Ectopic expression of Arabidopsis FUL in Brassica juncea prevented differentiation of lignified cells in the valve margins, leading to pod shatter resistance (Ostergaard et al., 2006). FILAMENTOUS FLOWER, YABBY3, and JAGGED can control the expression patterns of FUL and SHP in the valves and valve margin (Dinneny et al., 2005). Similar to FUL, REPLUMLESS also negatively regulates $S H P$ expression (Roeder et al., 2003).

Cultivated Brassica species include 3 diploids, Brassica rapa (AA genomes), Brassica nigra (BB), and Brassica oleracea (CC), and 3 allopolyploids, B. napus (AACC), B. juncea (AABB), and Brassica carinata (BBCC). The 3 diploid species were derived from an ancient hexaploid ancestor with a primary genome similar to that of $A$. thaliana (Lysak et al., 2005). The 3 allopolyploids originated from spontaneous hybridization between 2 of the 3 diploids (U, 1935). Each of the 3 allopolyploids contains 2 sets of homologous chromosomes, which originated from the 2 diploid species. Since the genome of Brassica species underwent a triplication event as well as other rearrangements during evolution, their genomes are more complex and divergent than those of $A$. thaliana (Lukens et al., 2003; Town et al., 2006). During genomic evolution in the Brassica species, homologous genes may have changed in function or become pseudo-genes after the 2 related diploid genomes fused and genetically recombined (Udall and Wendel, 2006; Chen and $\mathrm{Ni}, 2006$ ). Indeed, change and rearrangement of homologous chromosomes have been demonstrated in B. napus (Udall et al., 2004). In the 3 Brassica allopolyploid genomes, functional changes in some homeologous genes may have also occurred.

In $A$. thaliana, FUL regulates valve margin elongation of the silique and promotes cell expansion in the valve, inhibiting pod shattering (Ferrandiz et al., 2000; Liljegren et al., 2004). Plant FUL genes have been shown to be involved in pod shattering, including FUL in Arabidopsis and MADSB in Sinapis alba (Chandler et al., 2005; Ostergaard et al., 2006). Ectopic expression of the $S$. alba MADSB gene, which is homologous to AtFUL, improved pod shatter resistance in B. napus (Chandler et al., 2005). Ectopic expression of the Arabidopsis FUL gene in $B$. juncea also prevented pod shattering (Ostergaard et al., 2006). Homologs of Arabidopsis $F U L$ may also play an important role in pod development in B. napus.

As an allopolyploid crop, B. napus contains the $\mathrm{A}$ and $\mathrm{C}$ genomes, originating from B. rapa (A) and B. oleracea (C). Functional analysis of homeologs from the $\mathrm{A}$ and $\mathrm{C}$ genomes is of considerable importance for molecular manipulation of oilseed rape. In this study, we identified and examined $F U L$ homologs in B. napus, B. rapa, and B. oleracea. Based on sequence differences of $F U L$ genes from the $\mathrm{A}$ and $\mathrm{C}$ genomes, primers that could be used to distinguish $F U L$ homeologs were developed. Using these homeolog-specific primers, reverse transcription (RT)-polymerase chain reaction (PCR) analysis showed that expression of FUL from the A genome was higher in pod shatter-resistant varieties than in susceptible varieties. This expression level divergence may be applied to select pod shatter-resistant lines in oilseed rape-breeding programs.

\section{MATERIAL AND METHODS}

\section{Plant materials}

A total of 80 varieties of $B$. napus were evaluated for pod shatter resistance. These materials included available open-pollinated cultivars and hybrids, which were all collected 
by the Rapeseed Heterosis Application group, Oil Crops Research Institute, and Chinese Academy of Agricultural Sciences (OCRI-CAAS, Wuhan, Hubei). Brassica rapa var. Shang-hai-qing, Xi-zhang-bai-cai 1, Xi-zhang-bai-cai 2, Xi-zhang-bai-cai 3, B. oleracea var. Ye-sheng-gan-lan, Hei-ye-ping-tou, Ping-xiang-pei-lan, and Xin-nong-ying-liu-huacai were kindly provided by Prof. Xiaoming Wu in Department of Rapeseed Germplasm of OCRI-CAAS.

\section{Assessment of pod shatter resistance}

To identify varieties with different pod shatter resistance levels, 80 varieties of $B$. $n a$ pus were screened by random impact test using a modified protocol (Wei et al., 2010). At the fully mature stage, pods were harvested and hung indoors for 4 weeks to balance their water content. Twenty well-developed intact pods were placed in a cylindrical container that was 19 $\mathrm{cm}$ in diameter and $14 \mathrm{~cm}$ in height, together with 12 steel balls that were $13 \mathrm{~mm}$ in diameter. The container was shaken on a horizontal shaker at $280 \mathrm{rpm}$. The number of cracked pods was counted after each minute of shaking and repeated for up to $10 \mathrm{~min}$. The broken pods were removed from the container after each counting. Five plants of each variety were tested and pods were collected randomly from each whole plant. The pod shatter resistance index was calculated using the following equation:

$$
(\mathrm{SRI})=1-\sum_{i=1}^{10} x_{i \times}(11-i) / 200
$$

where $X_{\mathrm{i}}$ is the number of broken pods at the $\mathrm{i}^{\text {th }}$ minute, $1 \leq \mathrm{i} \leq 10$.

\section{DNA extraction, RNA extraction, and cDNA synthesis}

Genomic DNA was isolated from leaves by cetyltrimethylammonium bromide extraction (Doyle and Doyle, 1990). Total RNA was isolated from various plant organs using Trizol reagent following manufacturer instructions (Invitrogen, Carlsbad, CA, USA) and was treated with RNase-free DNaseI (Qiagen, Hilden, Germany). Total RNA ( $2 \mu \mathrm{g}$ ) was reverse transcribed into cDNA using the M-MLV RTase cDNA Synthesis Kit (Takara, Shiga, Japan).

\section{Identification and characterization of BnaX.FUL cDNA and genomic DNA}

The nucleotide sequence of Arabidopsis AGL8 (AT5G60910) was used as query sequence to search against the EST database of B. napus in GenBank through BLASTN. Three ESTs (GenBank \#EE448759.1, \#EV092733.1, \#CD841921.1) were identified and then chosen as the template sequences for PCR primer design. A 695-bp homologous sequence was amplified from R2 silique cDNA using primers FUL-F-1 and FUL-R-1 (all primer sequences are listed in Table 1). To obtain the entire cDNA sequence, 5'- and 3'-rapid amplification of cDNA ends (RACE) was carried out. The primer for 5'-RACE was FUL-RACE-5 and for 3'-RACE 
was FUL-RACE-3. RACE was performed according to manufacturer instructions (Smart RACE cDNA Amplification kit, Clontech, Mountain View, CA, USA) with silique cDNA. According to sequences obtained from RACE, 2 primer pairs were designed to amplify the genomic DNA of the FUL gene from R1-1 and R2. The primers were FUL-F-2 and FUL-R-2, and FUL-F-3 and FUL-R-3. PCR was conducted using a $20-\mu$ L reaction system and consisted of $8.75 \mu \mathrm{L} \mathrm{ddH}_{2} \mathrm{O}, 2 \mu \mathrm{L}$ 10X LA PCR buffer, $4 \mu \mathrm{L} 2.5 \mathrm{mM}$ dNTPs, $2 \mu \mathrm{L} \mathrm{MgCl}_{2}(25 \mathrm{mM}), 1$ $\mu \mathrm{L}$ of each $10 \mathrm{mM}$ primer, $1 \mu \mathrm{L} 100 \mathrm{mM}$ DNA, and $0.25 \mu \mathrm{L}$ Takara LA Taq $(5 \mathrm{U} / \mu \mathrm{L})$. The amplification conditions were as follows: pre-denaturation at $94^{\circ} \mathrm{C}$ for $3 \mathrm{~min} ; 34$ cycles for $45 \mathrm{~s}$ at $94^{\circ} \mathrm{C}, 45 \mathrm{~s}$ at $50^{\circ} \mathrm{C}$, and $72^{\circ} \mathrm{C}$ for $3 \mathrm{~min}$; finally, an 8 -min extension at $72^{\circ} \mathrm{C}$. All PCR products were separated by electrophoresis on $1.2 \%$ agarose gels and were purified using the DNA Rapid Purification Kit (Omega Bio-Teck, Norcross, GA, USA). Purified products were cloned into the PMD18-T vector (Takara) and transformed into Escherichia coli strain DH5 $\alpha$. A total of 12 single colonies for each PCR were picked for sequencing using the ABI 3730 (Applied Biosystems, Foster City, CA, USA).

\begin{tabular}{llc}
\multicolumn{2}{c}{ Table 1. Primers used for PCR amplification in this study. } & Prime purpose \\
\hline Prime name & Prime sequence (5'-3') & FUL gene fragment PCR \\
\hline FUL-F-1 & AAAGAGACATGGGAAGGGGTA & FUL gene RACE \\
FUL-R-1 & GTTTGGTTCAATCAATGACGAT & \\
FUL-RACE-5 & CAATGACGATGTACCGCCGTTCTC & FUL gene DNA PCR \\
FUL-RACE-3 & GAGAAGAACACGGGTCAGCAAGAAG & \\
FUL-F-2 & TTATTTATGGGAAGGGGTAGGGT & FUL gene CDS PCR \\
FUL-R-2 & TTGTTTGTCTGATATAAATAGCGA & \\
FUL-R-3 & ATGATCGCTATTTATATTCAGACAA & A genome special primer \\
MQ-FUL-F & CGTTCTTGACATTGTAATTCCGT & C genome special primer \\
MQ-FUL-R & CGCGGATCCATGGGAAGGGGTAGGGT & Control \\
BrFUL-600-F & CCGCGGAATTCTCATACTTAGGTAATTTTACTCATT \\
BoFUL-600-F & GCCCAGTACTGCGTAATCG & \\
FUL-UTR-R & GCCCAGTACTGCGTAATCT & \\
BnActin-F & GTTCTTGACATTGTAATTCCGT & \\
BnActin-R & TCTGGCATCACACTTTCTACAACGAGC & \\
\hline
\end{tabular}

\section{Cloning of FUL homologs in B. rapa and B. oleracea}

Using cDNA from a developing pod as template, the open reading frame of each FUL homolog was amplified using primers MQ-FUL-F and MQ-FUL-R. PCRs consisted of 10.75 $\mu \mathrm{L} \mathrm{ddH}_{2} \mathrm{O}, 2 \mu \mathrm{L}$ 10X Ex Taq buffer, $2 \mu \mathrm{L} 2.5 \mathrm{mM}$ dNTPs, $2 \mu \mathrm{L} \mathrm{MgCl}_{2}(25 \mathrm{mM}), 1 \mu \mathrm{L}$ of each $10 \mathrm{mM}$ primer, $1 \mu \mathrm{L} 100 \mathrm{mM}$ cDNA, and $0.25 \mu \mathrm{L}$ Takara Ex Taq $(5 \mathrm{U} / \mu \mathrm{L})$. The amplification conditions were: pre-denaturation at $94^{\circ} \mathrm{C}$ for $5 \mathrm{~min} ; 34$ cycles for $30 \mathrm{~s}$ at $94^{\circ} \mathrm{C}, 30 \mathrm{~s}$ at $62^{\circ} \mathrm{C}$, $72^{\circ} \mathrm{C}$ for $1 \mathrm{~min} ; 72^{\circ} \mathrm{C}$ for $8 \mathrm{~min}$. After cloning and sequencing, sequence analysis was carried out using the DNAMAN software.

\section{Homeolog-specific PCR primer design}

Because the cDNAs of BnaA.FUL and BnaC.FUL shared a high level of sequence similarity, it was difficult to design primers for real-time quantitative RT-PCR. To perform semi-quantitative RT-PCR and allow comparison of expression levels of the homeologs, ho- 
meolog-specific markers that could distinguish the BnaA.FUL and BnaC.FUL genes based on sequence divergence were developed. Four single nucleotide polymorphisms (SNPs) in the coding sequences (CDS) between BnaA.FUL and BnaC.FUL were identified by sequence comparison. Based on these SNPs, primers were designed using a modified singlenucleotide amplified polymorphism procedure (Drenkard et al., 2000). For this procedure, an SNP-specific base was introduced at the 3 '-end of the forward primers. To improve the specificity and efficiency of genome-specific PCR, a " $C$ " to " $T$ " mismatched base was added at the third base to the 3 '-end of the primers. When the A genome-specific primers were used to amplify $F U L$ from the $\mathrm{C}$ genome, no amplification should occur because there were 2 mismatches in the 3'-end. However, when the $F U L$ from the A genome was amplified using these primers, the amplification should be successful because there was only 1 base mismatch at the third base to the 3 '-end. Therefore, it was possible to detect variation in polymorphic sites within FUL genes of Brassica A and C genomes by PCR. The genome-specific FUL gene primers were BrFUL-600-F and FUL-UTR-R for BnaA.FUL and BoFUL-600-F and FULUTR-R for BnaC.FUL (sequences listed in Table 1).

\section{RT-PCR analysis of BnaX.FUL}

Using homeolog-specific PCR primers BrFUL-600-F and FUL-UTR-R for BnaA.FUL and BoFUL-600-F and FUL-UTR-R, organ-specific expression of BnaC.FUL was detected. Various tissues including root, stem, leaf, bud, petal, developing silique, valve, stamen, and developing seed were collected at various plant developmental stages. The PCR conditions were as follows: pre-denaturation at $94^{\circ} \mathrm{C}$ for $3 \mathrm{~min} ; 34$ cycles for $30 \mathrm{~s}$ at $94^{\circ} \mathrm{C}, 30 \mathrm{~s}$ at $53^{\circ} \mathrm{C}$, and $72^{\circ} \mathrm{C}$ for $1 \mathrm{~min}$; finally, an 8 -min extension at $72^{\circ} \mathrm{C}$. For all RT-PCRs, the housekeeping gene BnActin (B. napus actin, AF1118122), as an internal gene control, was amplified using primers BnActin-F and BnActin-R (Table 1). The agarose gel images were analyzed using the Bio-Rad Image Lab 3.0 software (Hercules, CA, USA).

\section{RESULTS}

\section{Screening for varieties with different levels of pod shatter resistance}

To examine the regulation of pod shatter resistance in $B$. napus, we first examined the pod shatter phenotype of many varieties and identified varieties that were very resistant or very susceptible to pod shattering. Analysis of 80 B. napus varieties showed that the SRI varied widely among varieties, ranging from $0.003-0.710$, with an average value of 0.182 (Table S1). Sixty-four varieties belonged to the susceptible shatter category, with SRI values below 0.300 , while 15 varieties showed medium resistance with SRI values from 0.300-0.500; these values were considered as intermediate shatter resistance (Figure 1). Only 1 variety, R1-1, showed an SRI over 0.500 , and was considered to be in the high shatter-resistant category. Based on these results, 2 extreme varieties were selected for further study, the resistant variety R1-1, with an SRI of 0.710, and the susceptible variety R2, with an SRI of 0.020 (Figure 2). 


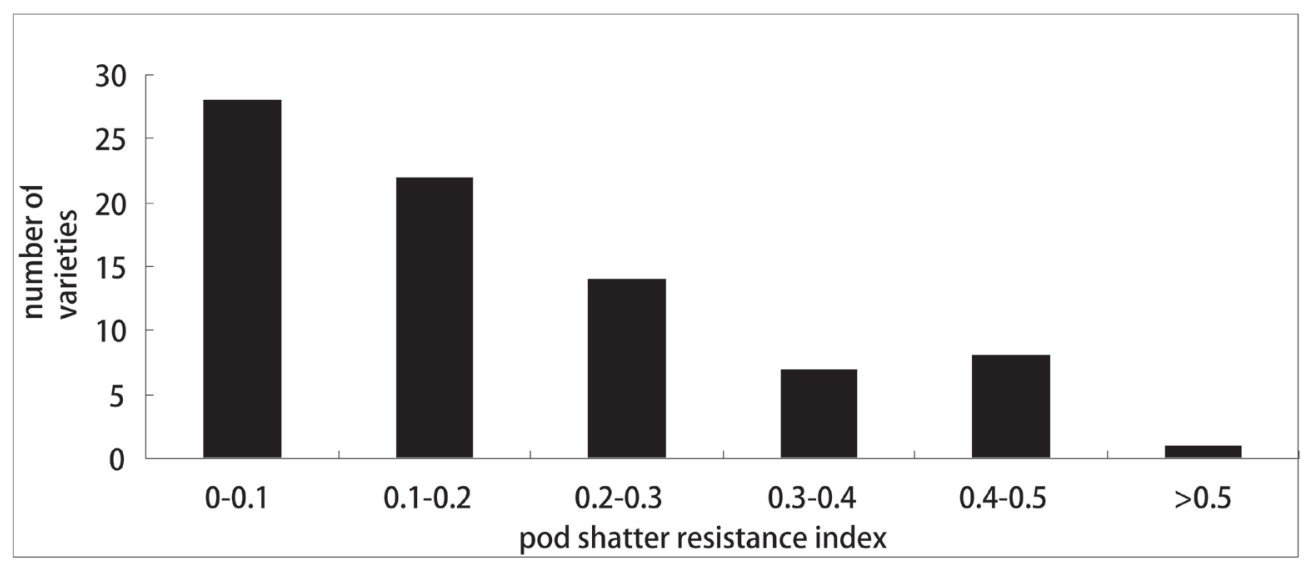

Figure 1. Distribution of pod shatter resistance indices in 80 varieties.
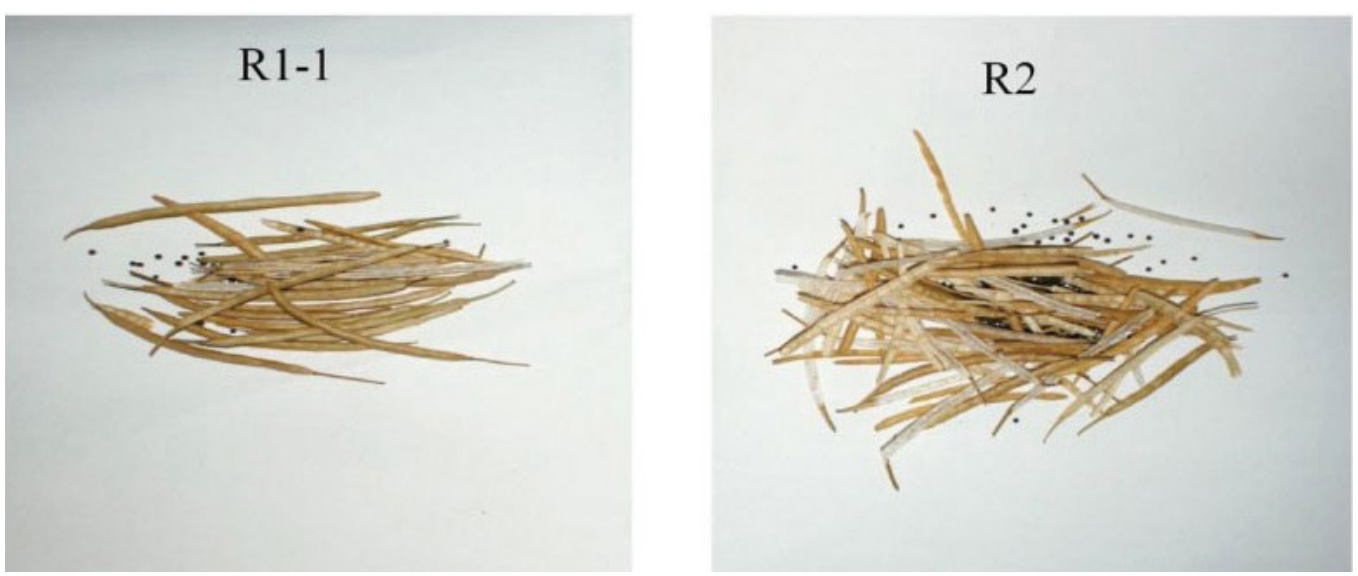

Figure 2. Pod shattering after random impact test using mature pods from resistant R1-1 and susceptible R2.

\section{Cloning and sequence analysis of FUL homeologs in Brassica}

With 5'- and 3'-RACE, 3 full-length cDNAs were identified in R1-1, whereas 7 fulllength cDNAs were found in R2, including the 3 identified in R1-1. These cDNAs showed high sequence similarity to $A t F U L$, with identity over $93 \%$, respectively, and thus were considered to be B. napus FUL homologs, referred to as BnaX.FUL.x. To assign the BnaX.FUL.x genes to $B$. napus genomes (A or $\mathrm{C}$ in the AACC allopolyploid), we next amplified the sequences of the B. napus ancestral species B. rapa (A genome) and B. oleracea (C genome). Sequence analysis of PCR products demonstrated that $3 F U L$ homeologs were present in $B$. rapa and 4 were present in $B$. oleracea. According to standard gene nomenclature (Ostergaard and King, 2008), the cDNAs identified in B. napus were named BnaA.FUL.a, BnaA.FUL.b, BnaA.FUL.c and BnaC.FUL.a, BnaC.FUL.b, BnaC.FUL.c, and BnaC.FUL.d based on their sequence similarity to the loci in $B$. rapa and B. oleracea, respectively. Sequence alignment 
analysis showed that the cDNAs shared high sequence similarity. There was only 1-3 nucleotide difference among homologs within the same genome. The difference among homologs between genomes, however, was more diverse with a clear 7-bp deletion in BnaA.FUL at the 3'-end compared to BnaC.FUL.

The CDS of FUL homeologs in these 3 Brassica species were highly conserved. Sequence alignments showed that the sequence at the 5 '-end was more conserved than the 3 '-end. In the coding regions, mismatches, including transitions and 7 deletions, were observed, and all of these changes resulted in amino acid substitutions between BnaA.FUL and BnaC.FUL. DNA sequence analysis showed that the BnaA.FUL and BnaC.FUL in the B. napus genome both consisted of 7 introns and 8 exons (Figure 3). Sequence alignment of the complete genes revealed high similarity between the $2 F U L$ homeologs, with over $98 \%$ identity. Some mismatches were found in the introns, particularly in the first and sixth introns.

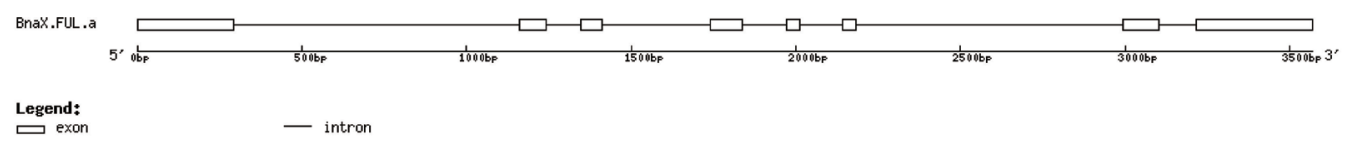

Figure 3. Structure of BnaX.FUL homeologs.

The deduced protein sequence of the BnaC.FUL protein contained 242 amino acids, while BnaA.FUL only contained 241 amino acids because of the 7-bp deletion of the CDS sequence. The 7-bp deletion led to a frameshift mutant, introducing premature termination for the CDS of amino acids in BnaA.FUL (Figure 4). Analysis using the Conserved Domain Database (http://www.ncbi.nlm.nih.gov/cdd) showed that both of the predicted proteins contained a typical MADS-box and K-box, which were highly conserved in FUL homeologs. To compare differences in the protein sequences, multiple alignment of amino acid sequences revealed that the 7 FUL proteins shared 98\% homology (Figure 4). In the MADS-box and Kbox domain, the sequences were highly conserved. A homology tree revealed that the amino acid sequence from the same genome was more closely related to each other and clustered together, while BnaA.FUL.c was more closely related to BnaC.FUL than BnaA.FUL.a and BnaA.FUL.b (Figure 5).

\section{Development of homeolog-specific markers for BnaX.FUL genes}

The specificity of homeolog-specific primers for performing semi-quantitative RTPCR was confirmed using genomic DNA of B. napus, B. rapa, and B. oleracea as templates. When the A genome-specific primers were used, the expected fragment was obtained from $B$. napus and B. rapa, whereas no amplified product was obtained from B. oleracea (Figure 6). Similarly, using the $\mathrm{C}$ genome-specific primers, 1 fragment was amplified from $B$. napus and B. oleracea, but no amplification occurred for B. rapa. Therefore, these fragments are specific markers for $F U L$ homeologs from A and $\mathrm{C}$ genomes and are suitable for expression analysis of different $F U L$ homeologs in B. napus. 


\begin{tabular}{|c|c|c|}
\hline $\begin{array}{l}\text { BnaA.FUL.a } \\
\text { BnaA.FUL.b }\end{array}$ & $\begin{array}{l}\text { NGRGRVQL KRI ENKI NRQVTFSKRRSGL L KKAHEI SVLCD } \\
\text { NGRGRVQLKRI ENKI NRQVTFSKRRSGL L KKAHEI SVLCD }\end{array}$ & $\begin{array}{l}40 \\
40\end{array}$ \\
\hline $\begin{array}{l}\text { BnaA.FUL. D } \\
\text { BnaA.FUL.c }\end{array}$ & NGRGRVQL KRI ENKI NRQVTFSKRRSGLL KKAHEI SVLCD & 40 \\
\hline BnaC.FUL.a & NGRGRVQL KRI ENKI NROVTF SKRRS GL L KKAHEI SVLCD & 40 \\
\hline BnaC.FUL.b & NGRGRVQL KRI ENKI NRQVTFSKRRS GL L KKAHEI SVLCD & 40 \\
\hline BnaC.FUL.c & NGRGRVQL KRI ENKI NRQVTFSKRRS GL LKKAHEI SVLCD & 40 \\
\hline BnaC.FUL.d & NGRGRVQL KRI ENKI NRQVTFSKRRS GL LKKAHEI SVLCD & 40 \\
\hline Consensus & rgrgrvql kri enki nrqvtfskrrsgl I kkahei svl c & \\
\hline BnaA.FUL.a & LVVFSSKGKLFEYSTDSSNERI LERYDRYLYSDKC & 80 \\
\hline BnaA.FUL.b & AEVAL VAFSSKGKLFEYSTDSSNERI LERYDRYLYSDKQL & 80 \\
\hline BnaA.FUL.c & AEVALVVFSSKGELFEYSTDSSNERI LERYDRYLYSDKQL & 80 \\
\hline BnaC.FUL.a & ILVVFSSKGKLFEYSTDSSNERI LERYDRYL & 80 \\
\hline C.FUL.b & AEVAL VVF SSKGKLFEYSTDSSMERI LERYDRYL & 80 \\
\hline BnaC.FUL.C & AEVALVVFSSKGKLFEYSTDSSNERI LERYDRYLYSDKQL & 80 \\
\hline BnaC.FUL.d & AEVALVVFSSKGKLFEYSTDSSMERI LERYDRYLYSDKC & 80 \\
\hline Consensus & aevalv fsskg ifeystdsstrerilerydrylysdkg & \\
\hline FUL.a & VGRDI SQSENVVLEHAKL KARVEVLEKNKRNF'NGEDLDS & 120 \\
\hline BnaA.FUL.b & VGRDI SQSENVVLEHAKLKARVEVLEKNKRNF NGEDLDS & 120 \\
\hline BnaA.FUL.c & VGRDI SQSENVVLEHAKL KARVEVLEKNKRNF NGEDLDSLL & 120 \\
\hline BnaC.FUL.a & VGRDI SQSENVVLEHAKLKARVEVLEKNKRNF NGEDLDS & 120 \\
\hline C.FUL.b & VGRDI SQSENV'ALEHAKLKARVEVLEKNKRNF NGEDLDSLL & 120 \\
\hline BnaC.FUL.c & VGRDI SQSENVVLEHAKL KARVEVLEKNKRNF NGEDLDS & 120 \\
\hline BnaC.FUL.d & VGRDI SQSENVVLEHAKL KARVEVLEKNKRNF NGEDLDS & 120 \\
\hline Consensus & vgrdi sqsenw $\overline{\text { Iehakl karvevleknkrnf rgedl } c}$ & \\
\hline L.a & SI KELQSLEHQLDAAI KSI RSRKNQANFESI SALQKKL & 160 \\
\hline L.b & SI KELQSLEHQL DAAI KSI RSRKNQANFESI SALC & 160 \\
\hline BnaA.FUL.c & SLKELQSLEHQLDAAI KSI RSRKNQANFESI SAL QKKDK & 160 \\
\hline BnaC.FUL.a & SI KELQSLEHQLDAAI KSI RSRKNQANFESI SALQKKD & 160 \\
\hline L.b & SLKELQSLEHQLDAAI KSI RSRKNQAMFESI SAL & 160 \\
\hline BnaC.FUL.C & SLKELQSLEHQLDAAI KSI RSRKNQAMFESI SALC & 160 \\
\hline BnaC.FUL.d & SLKELQSLEHQLDAAI KSI RSRKNQAMFESI SALQKKDK & 160 \\
\hline Consensus & s kel qsl ehql daaiksi rsrknqanfesisal c & \\
\hline JL.a & LQDHNNILL KKI KEKEKEKNT GQQEGQL】 QCSNNSSVLQP & 200 \\
\hline L.b & LQDHNNTLLKKI KEKEKEKNTGQQEGQLI QCSNNSSVLQP & 200 \\
\hline A.FUL.C & LQDHNNTLLKKI KEKEKEKNTGQQEGQLI QCSNNSSVLQP & 200 \\
\hline BnaC.FUL.a & LQDHNNTLLKKI KEKEKEKNT GQQEGQLI QCSNNSSVLQP & 200 \\
\hline BnaC.FUL.b & LQDHNNTLLKKI KEKEKEKNT GQQEGQLI QCSNNSSVLQP & 200 \\
\hline L.C & LQDHNNTLLKKI KEKEKEKNT GQQEGQ\$ QCSNNSSVLQP & 200 \\
\hline C.FUL.d & LQDHNNTLLKKI KEKEKEKNT GQQEGQL & 200 \\
\hline Consensus & Iqdhnnt I Ikki kekekeknt gqqegq i qcsnns & \\
\hline A.FUL.a & QYCVT ASRDGL VERV VGENGGGSSLI EPNSLLPAWN IRRSN & 240 \\
\hline JL.b & QYCVTASRDGL VERVVGENGGGSSLI EPNSPLPAMMLLLQN & 240 \\
\hline JL.C & QYCVTSSRDGL VERVGGENGGTSSLI EPNSLLPAMMLRP'N & 240 \\
\hline BnaC.FUL.a & QYCVTSSRDGL VERVGGENGGTSSLI EPNSLLPAMMLRPT & 240 \\
\hline C.FUL.b & QYCVTSSRDGL VERVGGENGGTSSLI EPNSLLPAMMLRPT & 240 \\
\hline C.FUL.C & QYCVTSSRDGL VERVGGENGGTSSLI EPNSLLPAUMLRPT & 240 \\
\hline C.FUL.d & QYCVTSSRDGL VERVGGENGGTS & 240 \\
\hline sus & qycvt srdglverv gengg ssli epns lpaum & \\
\hline A.FUL.a & & 241 \\
\hline BnaA.FUL.b & & 241 \\
\hline .FUL.C & & 241 \\
\hline E.FUL.a & $\mathbf{T}$ & 241 \\
\hline BnaC.FUL.b & $\mathbf{T}$ & 241 \\
\hline BnaC.FUL.C & $\mathbf{T}$ & 241 \\
\hline BnaC.FUL.d & $\begin{array}{lll}T \\
T\end{array}$ & 241 \\
\hline
\end{tabular}

Figure 4. Sequence analysis of the 7 BnaX.FUL proteins. Dark shading revealed $100 \%$ sequence identity, white shading revealed above $75 \%$ sequence identity, and gray shading revealed above $50 \%$ sequence identity. Sequence alignment was carried out using the DNAMAN software. 


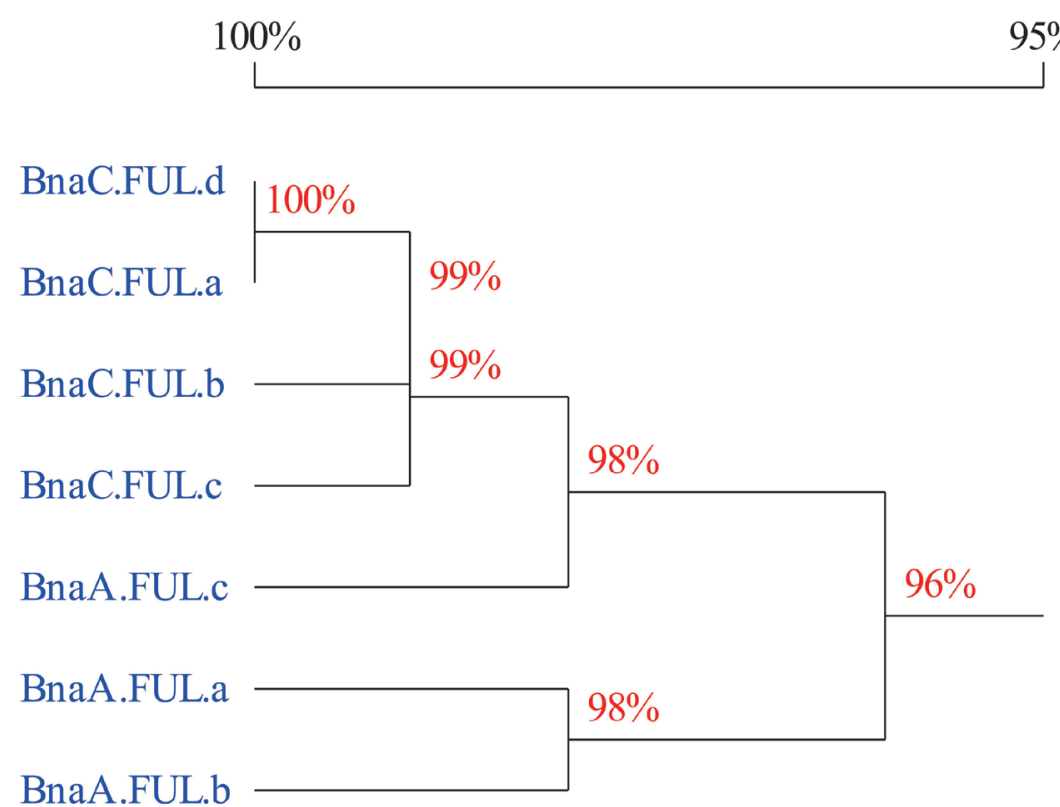

Figure 5. Homology tree of the 7 BnaX.FUL proteins. The bootstrap values are shown beneath each homology tree.

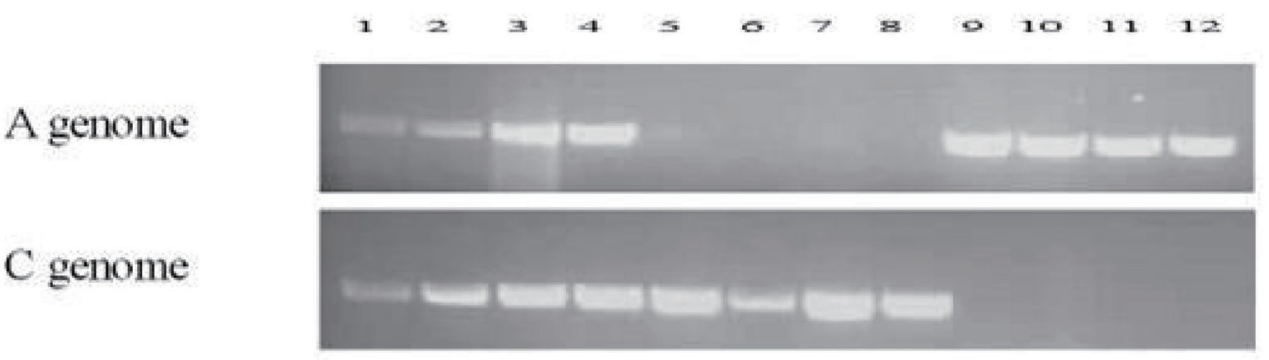

Figure 6. Amplification of FUL genes from different Brassica genomes using homeolog-specific primers. Lanes 1-4, B. napus R1, Zh11, R2, 8908B; lanes 5-8, B. oleracea Ye-sheng-gan-lan, Hei-ye-ping-tou, Ping-xiang-pei-lan, Xin-nong-ying-liu-hua-cai; lanes 9-12, B. rapa Shang-hai-qing, Xi-zhang-bai-cai 1, Xi-zhang-bai-cai 2, Xi-zhangbai-cai 3 .

\section{Expression analysis of BnaX.FUL}

To determine the expression pattern of BnaX.FUL in cultivars with different pod shatter resistance levels, semi-quantitative RT-PCR was performed using the homeologspecific primers with cDNAs from R1-1 and R2 as templates. This showed that the BnaX. FUL genes were expressed in a wide range of organs, including the stem, leaf, bud, petal, developing silique, and valve, but not in the root, stamen, and seed. Both resistant and susceptible varieties showed the same pattern of tissue specificity (Figure 7), but 1 expression difference was detected between R1-1 and R2. In the susceptible variety R2, both BnaA.FUL and BnaC.FUL were expressed, with the highest expression in developing silique, whereas in the resistant variety R1-1, only BnaA.FUL was expressed and high expression was detected in 
the young flower bud, developing pod, and valve. However, BnaC.FUL was not expressed in any tissue of R1-1 (Figure 7).

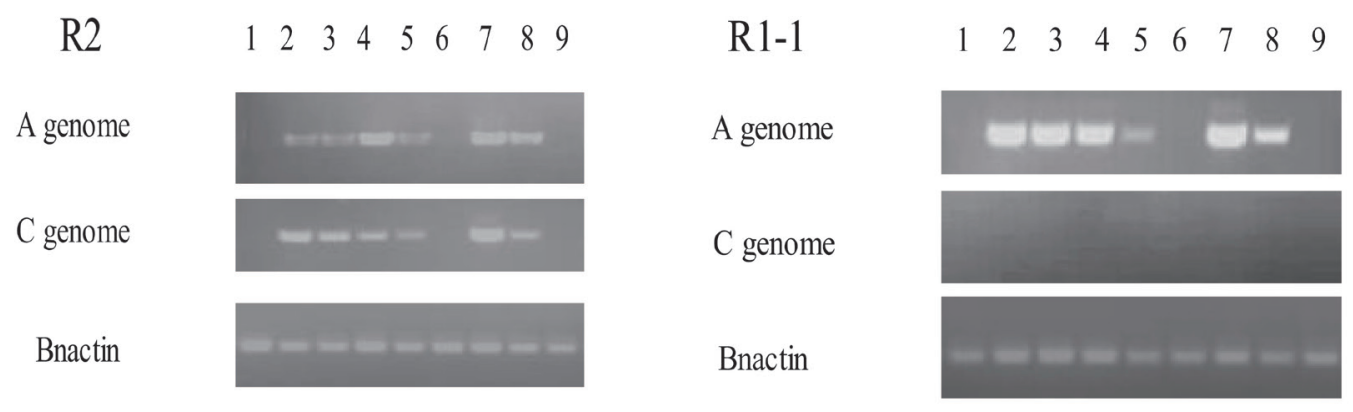

Figure 7. Expression of BnaX.FUL in R1-1 and R2 by RT-PCR. Lane 1, Root; lane 2, stem; lane 3, leaf; lane 4, young flower bud; lane 5, petal; lane 6, stamen; lane 7, developing pod; lane 8, valve; lane 9, developing seed.

To examine the correlation between the expression levels of BnaX.FUL and pod shatter resistance, we further analyzed 2 moderately resistant varieties Zh11 and R1, both with an SRI above 0.450 and 1 more susceptible variety $8908 \mathrm{~B}(\mathrm{SRI}=0.003)$, using semi-quantitative RT-PCR with silique cDNA samples. The results clearly classified the varieties into 3 groups (Figure 8). In the first group, the highly resistant variety R1-1, only BnaA.FUL was expressed at a high level, whereas no BnaC.FUL expression was detected. In the second group, the moderately resistant varieties Zh11 and R1, both BnaA.FUL and BnaC.FUL, were expressed. In the third group, the susceptible varieties R2 and 8908B, both homeologs were expressed, but expression of BnaA.FUL was significantly lower than that in the other groups. The expression level of BnaC.FUL was the same across the varieties except for R1-1.

\section{A genome}

\section{R1-1 R1 Zh11 R2 8908B}

\section{C genome}

\section{Bnactin}

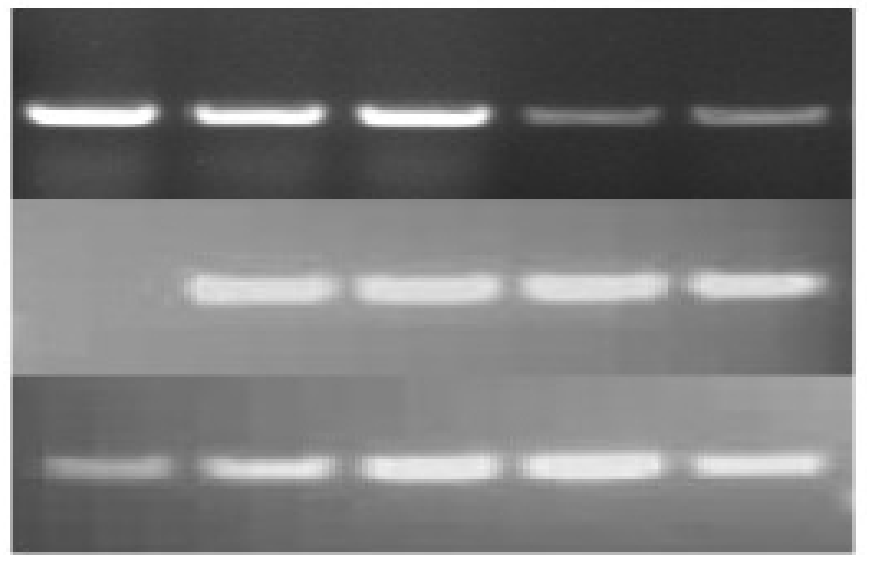

Figure 8. Semi-quantitative RT-PCR analysis of BnaX.FUL in siliques of Brassica napus varieties with different shatter resistance levels. 


\section{DISCUSSION}

The genetic improvement of pod shatter resistance in B. napus has been limited by lack of resistant germplasms and a reliable assessment method (Wang et al., 2007). Here, we screened for pod shatter resistance in a wide range of $B$. napus varieties using an improved random impact test (Wei et al., 2010), and identified a highly resistant variety R1-1 after at least 3 years of testing (data not shown).

Previous studies identified a number of genes that positively or negatively regulate pod shattering, including IND, ALC, SHP, REPLUMLESS, and FUL, in Arabidopsis. Except for the FUL gene, these homolog genes showed no expression pattern variation. FUL was involved in valve margin elongation in Arabidopsis siliques (Liljegren et al., 2000). Ectopic expression of the Arabidopsis FUL gene in B. juncea prevented pod shattering and showed that valve margin specification was conserved between Arabidopsis and Brassicas (Ostergaard et al., 2006). In our study, to characterize the molecular differences associated with pod shatter resistance level, the FUL homeologs in B. napus, B. rapa, and B. oleracea were cloned and analyzed. The sequences of BnaX.FUL were highly conserved, as expected. Using the genome sequence of B. rapa (http://brassicadb.org) and GenBank database (http://ncbi. nlm.nih.gov) and a standard BLAST search, some paralogs with very high sequence identity were found. Because homeologs were derived from the same ancestral gene, they are typically highly conserved, as observed for many genes in polyploid species (Wang et al., 2009; Huang and Brûlé-Babel, 2010).

Our results indicate that expression level differences of BnaX.FUL may affect pod shatter resistance. Both BnaA.FUL and BnaC.FUL were expressed in the same organs, showing high levels of redundancy. However, the expression level of BnaA.FUL differed between resistant and susceptible varieties. In the pod shatter-resistant variety, BnaA.FUL expression was significantly higher than that in the susceptible varieties. Additionally, in the high pod shatter-resistant variety R1-1, only BnaA.FUL was expressed in the detected organs, whereas the BnaC.FUL transcript was not detected in any organs. To avoid errors in measuring the expression level because of the sample or PCR technique, different cycles of PCR, including 30 and 40 cycles, were performed, and the results were consistent (data not shown). Thus, expression divergence of BnaA.FUL play a role in pod shatter resistance for different varieties.

To overcome genome instability and reproductive degeneration, it has been suggested that polyploid species tend to acquire diploid-like characteristics through massive gene loss, genomic reorganization through silencing, and unequal expression of homolog genes (Adams et al., 2003; Chen, 2007). Alterations in gene expression upon allopolyploidization have been reported in many plant species, including cotton, wheat, Arabidopsis, Senecio, Brassica, and Tragopogon (Buggs et al., 2010). In B. napus, differential expression of homeologs was previously observed for the ALC (Hua et al., 2009) and biotin carboxylase (Li et al., 2011) genes. $\mathrm{Xu}$ et al. (2009) found that in resynthesized B. napus, the proportions of C-genome-specific gene silencing were significantly greater than those of A-genome-specific gene silencing. We found that BnaC.FUL expression was silenced, supported their results, but was different from the results for BnaX.ALC described by Hua et al. (2009) in terms of genome bias of gene silencing. There is no documentation on genome bias of gene silencing in Brassicas, and it is not clear whether gene silencing in B. napus is biased towards a specific genome as only a small number of genes was investigated. None of the above expression alterations of homologous genes, however, were correlated with phenotypic differences. 
Alterations in the expression of homologous genes should lead to functional differences, as was recently shown for BnGPAT4 in B. napus (Chen et al., 2011). In this study, the absence of BnaC.FUL expression in the resistant variety did not occur in moderately resistant varieties. Instead, a difference in the expression levels of BnaA.FUL was observed across all varieties. Higher expression of BnaA.FUL was correlated with higher shatter resistance levels in the 5 tested varieties. Although in the highly resistant variety R1-1, silencing of BnaC.FUL appeared to play a role in enhanced resistance, the expression level of this homeolog did not differ in moderately resistant and susceptible varieties. It is more likely that the enhanced expression of BnaA.FUL was responsible for pod shatter resistance in resistant varieties rather than the depressed expression of BnaC.FUL in R1-1. In general, variations in pod shatter resistance in B. napus were limited; however, there was wide variation in B. rapa (Kadkol et al., 1985). Thus, the expression levels of $F U L$ from the A genome that were correlated with pod shatter resistance may explain this observation. In allopolyploids, cis-regulatory elements and trans-action effects may alter the expression of homeologous genes. Functional divergence is often caused by changes in cis-regulatory elements (Wittkopp et al., 2004; Wang et al., 2006; Chen, 2007). We deduced that the cis-regulatory elements in the sequence of BnaX.FUL may be different, causing functional divergence of BnaX.FUL. The mechanisms behind the expression divergence of homeologous $F U L$ loci and their effects on phenotypic variation require further investigation.

In most winter rapeseed planting areas, such as in China, the trend of combine harvesting of rapeseed will increase as labor resource shifting to cities from agricultural lands. Breeding rapeseed varieties with high pod shatter resistance is very important, but the number of pod shatter-resistant rapeseed germplasms is low. Thus, BnaA.FUL may be very useful for developing rapeseed varieties that are suitable for mechanical harvesting both through a transgenic approach and through marker-assistant selection using the expression level as a molecular marker.

In summary, 80 rapeseed varieties were screened for pod shatter resistance and some resistant varieties were identified. The homologous FUL genes in B. napus, B. rapa, and $B$. oleracea were cloned and characterized. SNP primers that could distinguish FUL gene loci from the different genomes of $B$. napus allowed for examination of the expression levels of BnaA.FUL and BnaC.FUL and revealed differences between resistant and susceptible varieties in B. napus. Importantly, expression levels of BnaA.FUL.a were correlated with the level of pod shatter resistance. The BnaX.FUL loci of different genomes showed sequence and function differentiation, which is an important consequence of allopolyploid evolution. The BnaA.FUL homeolog has great potential for the genetic improvement of oilseed rape, particularly for the development of lines suitable for mechanized harvesting.

\section{ACKNOWLEDGMENTS}

Research supported by the National Program on Key Basic Research Project ("973” Program, \#2011CB109302), the National High-Tech R\&D Program of China ("863" Program, \#2012AA101107) of the Ministry of Science and Technology of China, the Earmarked Fund for China Agriculture Research System (CARS-13), and the Hubei Agricultural Science and Technology Innovation Center. We are grateful to Dr. Yancheng Wen for his instructions on pod shatter resistance assessment and Ms. Hongyu An for her assistance with the assessment. 


\section{Supplementary material}

\section{REFERENCES}

Adams KL, Cronn R, Percifield R and Wendel JF (2003). Genes duplicated by polyploidy show unequal contributions to the transcriptome and organ-specific reciprocal silencing. Proc. Natl. Acad. Sci. USA 100: 4649-4654.

Bolle C, Schneider A and Leister D (2011). Perspectives on systematic analyses of gene function in Arabidopsis thaliana: new tools, topics and trends. Curr. Genomics 12: 1-14.

Buggs RJ, Elliott NM, Zhang L, Koh J, et al. (2010). Tissue-specific silencing of homoeologs in natural populations of the recent allopolyploid Tragopogon mirus. New Phytol. 186: 175-183.

Chai GH, Bai ZT, Wei F, King G J, et al. (2010). Brassica GLABRA2 genes: analysis of function related to seed oil content and development of functional markers. Theor. Appl. Genet. 120: 1597-1610.

Chandler J, Corbesier L, Spielmann P, Dettendorfer J, et al. (2005). Modulating flowering time and prevention of pod shatter in oilseed rape. Mol. Breed. 15: 87-94.

Chen ZJ (2007). Genetic and epigenetic mechanisms for gene expression and phenotypic variation in plant polyploids. Annu. Rev. Plant Biol. 58: 377-406.

Chen ZJ and Ni ZF (2006). Mechanisms of genomic rearrangements and gene expression changes in plant polyploids. Bioessays 28: 240-252.

Chen X, Truksa M, Snyder CL, El-Mezawy A, et al. (2011). Three homologous genes encoding sn-glycerol-3-phosphate acyltransferase 4 exhibit different expression patterns and functional divergence in Brassica napus. Plant Physiol. 155: 851-865.

Child R, Summers JE, Babij J, Farrentand JW, et al. (2003). Increased resistance to pod shatter is associated with changes in the vascular structure in pods of a resynthesized Brassica napus line. J. Exp. Bot. 54: 1919-1930.

Dinneny JR, Weige D and Yanofsky MF (2005). A genetic framework for fruit patterning in Arabidopsis thaliana. Development 132: 4687-4696.

Doyle JJ and Doyle JL (1990). Isolation of plant DNA from fresh tissue. Focus 12: 13-15.

Drenkard E, Richter BG, Rozen S, Stutius LM, et al. (2000). A simple procedure for the analysis of single nucleotide polymorphisms facilitates map-based cloning in Arabidopsis. Plant Physiol. 124: 1483-1492.

Ferrandiz C, Liljegren SJ and Yanofsky MF (2000). Negative regulation of the SHATTERPROOF genes by FRUITFULL during Arabidopsis fruit development. Science 289: 54-78.

Gu Q, Ferrándiz C, Yanofsky MF and Martienssen R (1998). The FRUITFULL MADS-box gene mediates cell differentiation during Arabidopsis fruit development. Development 125: 1509-1517.

Hua SJ, Shamsi IH, Guo Y, Pak H, et al. (2009). Sequence, expression divergence and complementation of homologous ALCATRAZ loci in Brassica napus. Planta 230: 493-503.

Huang XQ and Brûlé-Babel A (2010). Development of genome-specific primers for homoeologous genes in allopolyploid species: the waxy and starch synthase II genes in allohexaploid wheat (Triticum aestivum L.) as examples. BMC Res. 3: 140 .

Kadkol GP, Halloran GM and Macmillan RH (1985). Evaluation of Brassica genotypes for resistance to shatter. II.Variation in siliqua strength within and between accessions. Euphytica 34: 915-924.

Lewis MW, Leslie ME and Liljegren SJ (2006). Plant separation: 50 ways to leave your mother. Curr. Opin. Plant Biol. 9: $59-65$

Li ZG, Yin WB, Song LY, Chen YH, et al. (2011). Genes encoding the biotin carboxylase subunit of acetyl-CoA carboxylase from Brassica napus and parental species: cloning, expression patterns, and evolution. Genome 54: 202-211.

Liljegren SJ, Ditta GS, Eshed Y, Savidge B, et al. (2000). SHATTERPROOF MADS-box genes control seed dispersal in Arabidopsis. Nature 404: 766-770.

Liljegren SJ, Roeder AH, Kempin SA, Gremski K, et al. (2004). Control of fruit patterning in Arabidopsis by INDEHISCENT. Cell 116: 843-853.

Lukens L, Zou F, Lydiate D, Parkin I, et al. (2003). Comparison of a Brassica oleracea genetic map with the genome of Arabidopsis thaliana. Genetics 164: 359-372.

Lysak MA, Koch MA, Pecinka A and Schubert I (2005). Chromosome triplication found across the tribe Brassiceae. Genome Res. 15: 516-525.

Ostergaard L and King GJ (2008). Standardized gene nomenclature for the Brassica genus. Plant Methods 4: 10.

Ostergaard L, Kempin SA, Bies D, Klee HJ, et al. (2006). Pod shatter resistant Brassica fruit produced by ectopic expression of the FRUITFULL gene. Plant Biotechnol. J. 4: 45-51. 
Rajani S and Sundaresan V (2001). The Arabidopsis myc/bHLH gene ALCATRAZ enables cell separation in fruit dehiscence. Curr. Biol. 11: 1914-1922.

Roeder AH, Ferrandiz C and Yanofsky MF (2003). The role of the REPLUMLESS homeodomain protein in patterning the Arabidopsis fruit. Curr. Biol. 13: 1630-1635.

Salentijn EMJ, Pereira A, Angenent GC, Linden CG, et al. (2007). Plant translational genomics: from model species to crops. Mol. Breed. 20: 1-13.

Town CD, Cheung F, Maiti R, Crabtree J, et al. (2006). Comparative genomics of Brassica oleracea and Arabidopsis thaliana reveal gene loss, fragmentation, and dispersal after polyploidy. Plant Cell 18: 1348-1359.

Udall JA, Quijada PA and Osborn TC (2004). Detection of chromosomal rearrangements derived from homologous recombination in four mapping populations of Brassica napus L. Genetics 169: 967-979.

Udall JA and Wendel JF (2006). Polyploidy and crop improvement. Crop Sci. 46: 3-13.

Wang J, Tian L, Lee HS and Chen ZJ (2006). Nonadditive regulation of FRI and FLC loci mediates flowering time variation in Arabidopsis allopolyploids. Genetics 173: 965-974.

Wang J, Long Y, Wu B, Liu J, et al. (2009). The evolution of Brassica napus FLOWERING LOCUS T paralogues in the context of inverted chromosomal duplication blocks. BMC Evol. Biol. 9: 271.

Wang R, Ripley VL and Rakow GR (2007). Pod shatter resistance evaluation in cultivars and breeding lines of Brassica napus, Brassica juncea and Sinapis alba. Plant Breed. 126: 588-595.

Wei WH, Li YC, Wang LJ, Liu SY, et al. (2010). Development of a novel Sinapis arvensis disomic addition line in Brassica napus containing the restorer gene for Nsa CMS and improved resistance to Sclerotinia sclerotiorum and pod shattering. Theor. Appl. Genet. 120: 1089-1097.

Wittkopp PJ, Haerum BK and Clark AG (2004). Evolutionary changes in cis and transgene regulation. Nature 430: 85-88.

$\mathrm{Xu}$ Y, Zhong L, Wu X, Fang X, et al. (2009). Rapid alterations of gene expression and cytosine methylation in newly synthesized Brassica napus allopolyploids. Planta 229: 471-483. 